\title{
Rascher Therapieerfolg bei Bronchitis
}

- Mit einer Kombination aus Thymian- und Efeu- bzw. Primelextrakten lindert Bronchipret $^{\circledR}$ Bronchitisbeschwerden rasch und effektiv. Für eine schnelle Linderung der Beschwerden und für die Wiederherstellung der natürlichen Schutzmechanismen ist die Normalisierung der Schleimschutzschicht vorrangig. Die bei Atemwegsinfekten meist gestörte mukoziliäre Clearance der Bronchien wird durch Bronchipret ${ }^{\oplus}$ wieder hergestellt. Zähes, schwer lösliches Sekret normalisiert sich, vorher verengte Bronchien können sich entspannen, der Patient besser durchatmen.

In einem aktuellen Bericht über den Einsatz von Mukolytika bei Bronchitis erläutert Dr. Riedl-Seifert, Kassel, weshalb der Einsatz synthetischer Sekretolytika nicht unkritisch erfolgen sollte:,Die meisten Schleimlöser zerstören die Integrität des bronchialen Mukus und führen zur Verflüssigung. Doch das zu sehr verflüssigte Sekret lässt sich schlecht abhusten, da der ursprünglich zusammen- hängende Sekretfilm reißt." Die Pflanzenextrakte Thymian und Efeu bzw. Primel regulieren vielmehr die Überproduktion von zähem, festsitzendem Schleim und normalisieren das Sekret. Dadurch wird die Schutzfunktion des Sekretfilms wieder hergestellt. Bronchipret ${ }^{\circledast}$ löst festsitzenden Husten, entspannt die Bronchialmuskulatur und befreit die Bronchien. Dadurch wird die natürliche Schutzfunktion der Bronchialschleimhaut regeneriert. Die Inhaltsstoffe kombinieren sekretolytische, antiphlogistische und bronchospasmolytische Wirkungen und verschaffen dadurch rasche Linderung mit nachhaltigem Effekt. Mit einer Kombination aus Thymian- und Efeuflüssigextrakt (Saft) bzw. Thymian- und Primelwurzeltrockenextrakt (Tablette) vereint das Phytopharmakon sekretolytische, aber auch und vor allem antiphlogistische und bronchospasmolytische Wirkungen.

Nach Informationen von Bionorica SE, Neumarkt

\section{PAH-CHD bei Kindern: spezifische Therapie entscheidend}

— „Etwa die Hälfte der Fälle der pädiatrischen pulmonal-arterielle Hypertension (PAH) beruht auf einem angeborenen Herzfehler", sagte Dr. Siegrun Mebus aus München, auf der Jahrestagung der Deutschen Gesellschaft für pädiatrische Kardiologie (DGPK) in Weimar. Diese spezielle Form der $\mathrm{PAH}$, die PAH-CHD (pulmonal-arterielle Hypertonie bei angeborenen Herzfehlern) schränkt besonders in ihrer schwersten Ausprägung, dem Eisenmenger-Syndrom, die Prognose und Lebensqualität der Patienten stark ein.

Die komplexe Pathophysiologie der PAHCHD erläuterte Prof. Dr. Johannes Breuer, Bonn: Danach können die Druck- und Volumenbelastungen infolge angeborener Herzfehler nach initialer pulmonaler Endothelstörung die Gefäßobstruktion verursachen. Indikationsstellung und Therapiesteuerung bei PAH-CHD erfordern viel Erfahrung, besonders wenn komplexe kardiale Fehlbildungen vorliegen. Therapeutisch stehen neben Phosphodiesterase-Inhibitoren und
Prostanoiden die Endothelin-RezeptorAntagonisten mit ihren vasodilatativen und antiproliferativen Effekten zur Verfügung. Als einziges PAH-Medikament wird hier das orale Bosentan (Tracleer $\left.{ }^{\circledast}\right)$ in internationalen Guidelines empfohlen.

Das Eisenmenger-Syndrom basiert auf einem Shunt-Vitium mit großem Links-RechtsShunt. Im Verlauf der pulmonalvaskulären Erkrankung steigt der Lungengefäßwiderstand an und es kommt zur Shuntumkehr. Der dann vorliegende Rechts-Links-Shunt kann aus einem azyanotischen einen zyanotischen Herzfehler machen. Beim Eisenmenger-Syndrom ist nur Bosentan als spezifisches PAH-Medikament zugelassen und steht auch in pädiatrischer Darreichungsform zur Verfügung.

jset

Satellitensymposium "Standortbestimmung und Perspektive: PAH bei Kindern und Jugendlichen" anlässlich der 42. Jahrestagung der DGPK. Weimar, 3. Oktober 2010. Veranstalter: Actelion, Freiburg
Kortikoidsparender Effekt bei Asthma bronchiale

Wenn bei Kindern mit kortikoidpflichtigem allergischen Asthma gegen Hausstaubmilben-Allergene eine spezifische Immuntherapie mit ACAROID ${ }^{\circledR}$ eingesetzt wird, so benötigen sie weniger inhalative Kortikoide. Dies ist das Resultat einer randomisierten Studie von Zielen et al. mit 65 Kindern im Alter zwischen sechs und 17 Jahren über zwei Jahre [J Allergy Clin Immunol 2010; 126: 942-9]. Die zur Symptomkontrolle erforderliche minimale Fluticasonpropionat-Dosis konnte bei den Patienten der Studie unter ACAROID ${ }^{\circledR}$ im Vergleich zur Kontrollgruppe, die ausschließlich eine antiasthmatische Basisund Bedarfsmedikation erhielt, signifikant reduziert werden $(p<0,05)$. Die tägliche Kortikoiddosis konnte duch die zusätzliche Gabe der SCIT nach zwei Jahren mehr als halbiert werden.

Trotz geringerer Kortikoidgaben blieb das Asthma gut kontrolliert und die Lungenfunktion im morgendlichen Peakflow besserte sich sogar signifikant gegenüber der Kontrollgruppe. ACAROID ${ }^{\circledast}$ ist für Kinder ab sechs Jahre geeignet. Es ist nicht nur für seine gute Effektivität, sondern auch für seine Anwenderfreundlichkeit und gute Verträglichkeit bekannt.

Nach Informationen von Allergopharma, Reinbek

\section{Note "gut" für Kopflausmittel}

14 Kopflausmittel wurden von der Zeitschrift ÖKOTEST geprüft. Unter anderem wurde die Wirksamkeit, Pharmakologie, Unbedenklichkeit der Inhalts- und Hilfsstoffe sowie Umweltfreundlichkeit der Verpackung bewertet. Dabei wurde NYDA ${ }^{\oplus}$ mit der Note 2 ausgezeichnet. NYDA ${ }^{\oplus}$ Marktführer unter den deutschen Kopflauspräparaten - enthält zwei unterschiedlich visköse Dimeticone: ein dünnflüssiges, leicht flüchtiges und ein dickflüssiges, schwer flüchtiges. Die Dimeticonlösung dringt tief in das Atemsystem der Läuse, Larven und Eier ein und verdrängt den Sauerstoff. Das dünnflüssige Dimeticon verflüchtigt sich, dadurch dickt die Lösung ein und verschließt so das Atemsystem irreversibel. Die Kopfläuse und alle ihre Entwicklungsstadien ersticken auf diese Weise. Das physikalische Wirkprinzip macht Resistenzentwicklungen äußerst unwahrscheinlich.

Nach Informationen von Pohl-Boskamp, Hohenlockstedt 